Int. J. Electrochem. Sci., 14 (2019) $7133-7148$

\title{
High Temperature Applicable Separator by Using Polyimide aerogel/polyethylene Double-Layer Composite Membrane for High-Safety Lithium Ion Battery
}

\author{
Gunhwi Kim ${ }^{\#}$, Jinyoung Kim ${ }^{\#}$, Jongyeob Jeong, Daero Lee, Myeongsoo Kim, Sangrae Lee, \\ Seohyun Kim, Hyunju Lee, Haksoo Han* \\ Department of Chemical and Biomolecular Engineering, Yonsei University, 262 Seongsan-no, \\ Seodaemun-gu, Seoul 120-749, South Korea \\ E-mail: hshanpublication@gmail.com \\ ${ }^{\#}$ These two authors have equally contributed to this study
}

doi: $10.20964 / 2019.08 .18$

Received: 5 March 2018 / Accepted: 16 July 2018 / Published: 30 June 2019

To address the vulnerability of a conventional separator to high temperature, a new double-layer function of separator with a double-layer function is suggested for high-safety lithium-ion batteries that are used in harsh condition. The new composite separator was fabricated by coating thermally imidized polyimide aerogel (PIA) on a porous polyethylene (PE) membrane, with polyvinylidene fluoride (PVDF) as a binder, to improve the thermal stability of the separator. The PIA/PE double-layer composite performed very well, especially in terms of thermal stability. The newly suggested separator showed a near-zero thermal shrinkage rate compared to commercial PE separators, which are defective in having high levels of the same, and retained its structure up to $140{ }^{\circ} \mathrm{C}$. The PIA supporting layer showed hardly any change after heat treatment, and the PE layer performed its role as a shut-down layer perfectly, ensuring the safety of the new separator. These results prove that a PIA/PE separator can prevent batteries from exploding and overcharging. In addition, the PIA/PE separator also demonstrated excellent electrolyte uptake and electrolyte wettability to electrolyte. PIA/PE separator based coin cells exhibited outstanding cycling and rate performances, especially at high current rates, compared to coin cells with a standard PE separator. Therefore, the new PIA/PE separator is an ideal candidate for use in high-safety lithium-ion batteries at high temperatures, based on its excellent thermal and chemical stability.

Keywords: Polyimide aerogel; Separator; Composite; Lithium-ion battery; Safety; Thermal stability. 
(C) 2019 The Authors. Published by ESG (www.electrochemsci.org). This article is an open access article distributed under the terms and conditions of the Creative Commons Attribution license (http://creativecommons.org/licenses/by/4.0/). 